\title{
DibA: An Adaptive Broadcasting Scheme in Mobile Ad Hoc Networks
}

\author{
Dimitrios Liarokapis, Ali Shahrabi, and Andreas Komninos \\ \{Dimitrios.Liarokapis, A.Shahrabi, Andreas.Komninos\}@gcal.ac.uk \\ School of Engineering and Computing \\ Glasgow Caledonian University, UK
}

\begin{abstract}
Broadcasting is a vital communication mechanism in Mobile Ad Hoc Networks, as it is the key element for exchanging control packets to support some services such as management and routing protocols. Simple Flooding, which is mostly used as the broadcasting mechanism, is neither resource efficient nor reliable especially in high populated and dense networks. The Distance-Based $(D B)$ scheme is one of the schemes proposed to alleviate the Flooding effects. However, DB uses a constant pre-determined distance threshold value regardless of network dynamics. In this paper, we propose an adaptive Distance-Based (DibA) scheme, which locally determines the density of the network and adjusts the distance threshold of every rebroadcasting operation without relying on HELLO packets or GPS systems. Our simulation study shows that our proposed scheme achieves considerable performance improvement over $D B$ while the average value of transmission packets is not a function of network size.
\end{abstract}

\section{INTRODUCTION}

The use of Mobile Ad hoc Networks (MANETs) is critically important in situations where mobile nodes are required to communicate with each other without relying on any fixed infrastructure, such as access points. Communication between mobile or static nodes in battlefields or disaster areas cannot depend on fixed infrastructure, thus MANETs are the only option to support network operations [1]. When the receiver is placed within the transmission range of the sender node, communication is possible through a single-hop connection. Otherwise, as long as there is a multi-hop path that could connect the two nodes, the exchange of packets is possible [2].

Despite the unique characteristics of Mobile Ad Hoc Networks, there are also many common points with traditional wireless networks. Operations like DNS lookups, exchange of control packets and routing discoveries, need to broadcast information to all or part of the network nodes. Simple Flooding (SF) is currently the dominant broadcast mechanism.

In SF, upon reception of a broadcast packet the receiver will automatically retransmit it to all of its neighbouring nodes. Highly populated and dense networks are typical examples where SF has been found to perform poorly in terms of reliability and resource efficiency, causing the Broadcast Storm Problem [3].

Over the past few years many studies [4], [5], [6], [7], [8], [9], [10] have been conducted to develop broadcast mechanisms to alleviate the effects of SF. The focus of the early works was on the schemes where the mobile nodes make the rebroadcast decision based on fixed and preconfigured thresholds. The most common thresholds relate to the distance between sender and receiver (Distance Based scheme - DB), the number of duplicate packets received (Counter Based scheme - CB), and a fixed probability for rebroadcast (Probability Based scheme - PB) [3]. Despite the fact that these schemes have been shown to considerably improve the overall performance of the network, they have been found to depend highly on the combination of threshold selected, density and level of population. The degree of dependency is such that in certain network topologies SF performs better than these schemes [11].

Adaptive schemes have consequently been proposed to alleviate these dependencies. In such schemes the threshold used for the broadcast operation changes according to the local density of the network, within the transmission range of the sender (number of one hop neighbours) or within an expanded neighbourhood area (number of two hops neighbours). To determine the density of the network locally, these schemes exchange HELLO packets [13], [14], [15], [16], [17], [18], [19] or use a positioning system, e.g. GPS, [12]. This either introduces more overhead traffic to the network or demands the existence of expensive positioning systems.

In this paper we propose a novel Distance-based Adaptive (DibA) scheme which is based on the Distance based scheme but dynamically adjusts the 
distance threshold value independently for every rebroadcast operation without using any expensive positioning system or exchanging control packets.

The remainder of this paper is organised as follows. In Section 2 we overview related work. Our adaptive broadcast mechanism is introduced in Section 3. In Section 4 we explain the process of building a highly diverse network topology where the performance of adaptive schemes can be evaluated appropriately. The performance study is presented in Section 5. Finally, we make concluding remarks in Section 6.

\section{RELATED WORKS}

In this section the Distance-based scheme will be presented in detail, as our proposed scheme enhances it by modifying its algorithm in order to make it locally adaptive. Furthermore, we will discuss the general characteristics of other adaptive schemes and their adaptive methods.

\section{Distance-based scheme}

$\mathrm{DB}$ is a broadcast mechanism that uses the distance between sender and receiver to make the decision whether to rebroadcast or not. The signal power from the packet received is a parameter that can be used to calculate the distance. GPS can also be used for that purpose. The specific algorithm for DB is as follows [3]:

$\mathrm{S} 1$. When a broadcast message, $m s g$, is heard for the first time, initialize $d_{\min }$ to the distance of the broadcasting node. If $d_{\text {min }}<D$ (where $D$ is the distance threshold), proceed to S5. In S2, if $m s g$ is heard again, interrupt the waiting and perform S4.

$\mathrm{S} 2$. Wait for a random number of slots. Then submit $m s g$ for transmission and wait until the transmission actually starts.

$\mathrm{S} 3$. The message is on the air. The procedure exits.

S4. Update $d_{\min }$ if the distance to the host from which $m s g$ is heard is smaller. If $d_{\text {min }}<D$, proceed to S5. Otherwise, resume the waiting in $\mathrm{S} 2$.

S5. Cancel the transmission of $m s g$ if it was submitted in S2. The host is inhibited from rebroadcasting message. Then exit.

The distance threshold used in DB, is a parameter valued by default. It is fixed and does not change dynamically, except if an administrator or the mobile node user intervenes manually. That is also the major drawback of DB, as a static threshold may be appropriate for a network of specific density but it could cause poor network performance when the density greatly differs [11].

\section{Adaptive schemes}

Over the past few years, a growing number of studies have been trying to develop adaptive versions of DB. In order to achieve this, having the instantaneous knowledge of network configuration (in particular, the number of mobile nodes placed within the transmission range of each sender) is required. There have been only two methods, to best of our knowledge, used to determine that parameter so far.

The first mechanism makes use of Global Positioning Systems (GPS) [12]. Mobile nodes periodically exchange messages including their exact coordinates. When a mobile node receives these coordinates it can calculate the distance from its current position and decides if the transmitting node is placed inside the transmission radius. In case that is true, the node increases its neighbours counter and therefore it can determine the level of network density locally. The use of expensive positioning systems, as GPS, is the limitation of this approach.

According to the second mechanism [13], [14], [15], [16], [17], [18], [19] the mobile nodes need to periodically send HELLO packets to all their neighbouring nodes and consequently count the number of responses they receive to measure the local density. It is obvious that this approach introduces a significant amount of overhead traffic in the network that could negatively affect the overall network performance, especially in cases where the network is highly populated and already overwhelmed with other types of traffic. In addition, one also needs to decide on the frequency of this procedure to take place. It has to be remembered that although an increase in performance is the net result of introducing overhead (i.e. HELLO packets) and reducing overhead (i.e. fewer rebroadcasting), a frequent transmission of HELLO packets in static networks only increases the amount of overhead.

Although both mechanisms are adaptive, they also have significant drawbacks that occasionally could produce additional constraints. In the next section, we propose a novel broadcast algorithm which is not relying on any positioning system or introducing overhead traffic.

\section{DISTANCE-BASED ADAPTIVE SCHEME (DibA)}

In order for a broadcast scheme to perform adaptively without introducing any further constraints, information about the local density of network is required to enable a mobile node to decide whether or not to rebroadcast.

In our approach, we make use of Step 2 (S2) of the DB original algorithm presented above and make minor changes to Step 4 (S4). According to $\mathrm{DB}$ in S2, the receiving mobile node needs to wait for a random number of slots and remains in listening mode for duplicate broadcast packets. During that period of time, upon reception of a 
duplicate packet, it calculates the new distance and compares it with the distance threshold $D$.

We take advantage of this waiting period and calculate the number of duplicate packets received, using a simple counter which is initialized and increased in S4. The number of identical packets arriving at the mobile node is closely connected to the number of neighbouring nodes. Each time the value of the counter increases, the distance threshold is tuned according to a pattern that is introduced administratively. This pattern is a scaled "if" statement, where the distance threshold changes its value with dependence to the current counter value. The number of possible values for the distance threshold is a parameter that is set by default. The value of the distance threshold could change multiple times during the waiting period and every time a duplicate broadcast packet is received, the distance between sender and receiver is compared with the current value of the threshold. The details of DibA algorithm is presented below, where $D$ is the distance threshold, count is the counter described above, $D_{1}, D_{2} \ldots D_{n}$ are the predetermined threshold values and $c_{1}, c_{2} \ldots c_{n}$ are predetermined counter values.

$\mathrm{S} 1$. When a broadcast message $m s g$ is heard for the first time, initialize $d_{\min }$ to the distance of the broadcasting node and the count to 1 . If $d_{\text {min }}<D$ (where $D$ is the distance threshold), proceed to S5. In S2, if $m s g$ is heard again, interrupt the waiting, increase count by 1 and perform S4.

$\mathrm{S} 2$. Wait for a random number of slots. Then submit $m s g$ for transmission and wait until the transmission actually starts.

$\mathrm{S} 3$. The message is on the air. The procedure exits.

S4. Update $d_{\min }$ if the distance to the host from which $m s g$ is heard is smaller.

If count is less than $c_{1}$

then set $D_{1}$ to $D$,

else if count is less than $c_{2}$

then set $D_{2}$ to $D$,

else ...

else if count is greater than $c_{n}$

then set $D_{n}$ to $D$.

If $d_{\min }<D$, proceed to S5. Otherwise, resume the waiting in $\mathrm{S} 2$.

S5. Cancel the transmission of $m s g$ if it was submitted in S2. The host is inhibited from rebroadcasting message. Then exit.

DibA's primary goal is not to calculate accurately the number of neighbouring nodes, but to decide upon the density level of the network locally inside the transmission radius. This feature gives an extra advantage to our approach in comparison to other adaptive schemes.

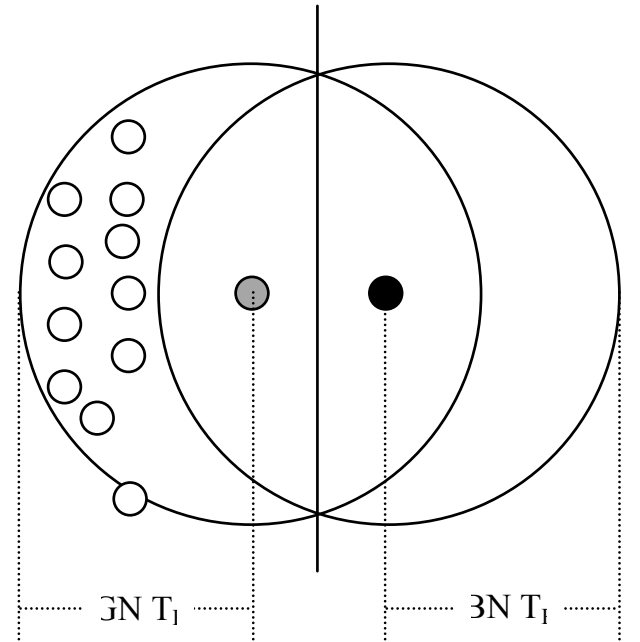

Figure 1. Diverse network topology.

Let us consider part of a network topology as shown in Figure 1. This is an extremely diverse topology as in the right part of the network only 1 node is placed. The left part of the network covers 12 nodes. The black node (BN) sends a broadcast message that is received by all its neighbours. In this occasion the only neighbour of $\mathrm{BN}$ is the grey node (GN). All nodes have the same transmission range $T_{R}$.

When we use one of the already existing adaptive schemes, GN will try to calculate the exact number of nodes inside the transmission radius. Either using GPS or HELLO packets, the end result of the calculation will be very close to 12 , the total of all white nodes (WN) and BN. As a result, GN will decide that the network is very dense locally and tune the distance threshold to be high, in order to rebroadcast only if it is placed at the edge of BN's transmission range. In case that the distance between $\mathrm{BN}$ and GN is not large enough to exceed the tuned distance threshold (Figure 2), GN will not rebroadcast. None of the WNs will receive the

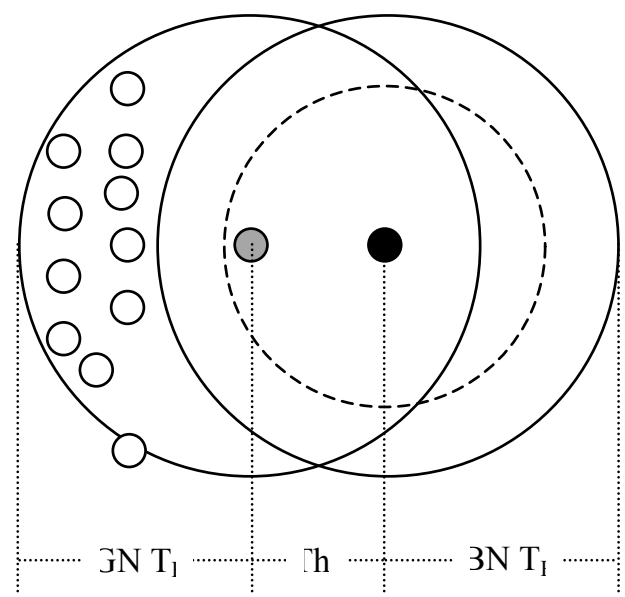

Figure 2: Existing Adaptive schemes. 


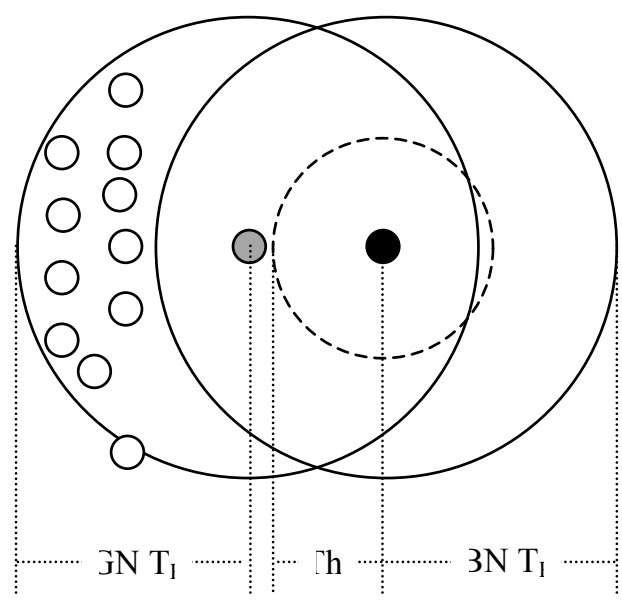

Figure 3. DibA.

broadcast packet.

In case that DibA is used as the broadcast scheme, after reception, GN will wait for a random period of time counting duplicate packets. As BN is the only neighbour that has broadcasted the packet, when GN exits the listening mode, the counter will be set to 1 , forcing the distance threshold to be assigned a very low value. It is highly possible at this point, as the threshold is very low, that GN is placed outside the dotted circle, as shown in Figure 3. As a result, GN will rebroadcast the packet and all WNs will receive it.

In this example, we have shown that knowing the exact number of neighbouring nodes is not always ideal when trying to decide upon the appropriate value for the distance threshold. DibA measures the level of local density, depending on duplicate receptions and not on the knowledge about the amount of neighbours. Thus, it is highly reliable for both normal and extremely diverse network topologies.

\section{BUILDING A DIVERSE NETWORK TOPOLOGY}

Most of studies [3], [4], [5], [6], [7], [8], [11], [13], [14], [15], [16], [17], [18], [19] are relying on a simple network topology consisted of nodes distributed nearly evenly in an area when studying the performance of a broadcast scheme. However, the performance of any adaptive scheme is more appropriately demonstrated when tested on a diverse network topology, where part or parts of the network significantly differ in mobile nodes population volumes. In this section, we present the implementation of an automatic mechanism that can be used to create this kind of topologies.

The simulation tool that we use for our experiments is NS-2.30. NS-2 offers a single tool for creating mobility files using the setdest command. The user has the options to select the length and width of the topology, the number of nodes, pause time, maximum and minimum speed

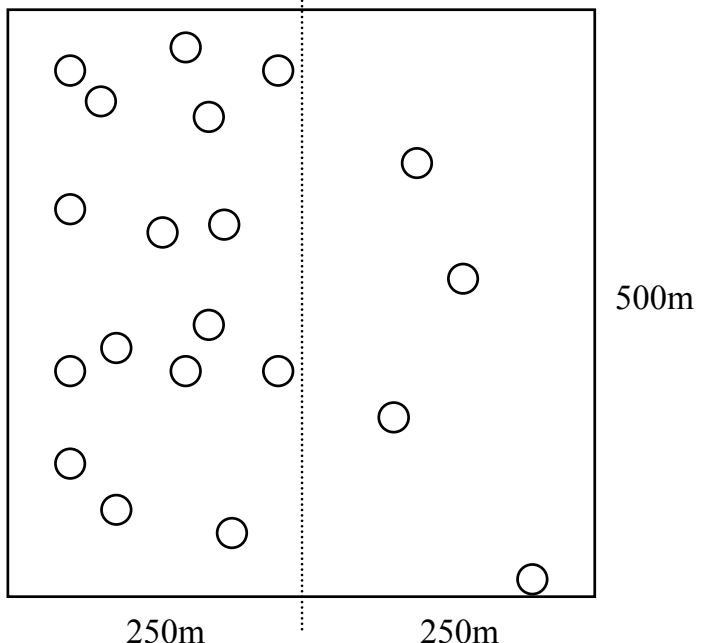

Figure 4. A sample diverse topology.

and simulation time. Unfortunately, setdest does not provide options to create more complex scenarios. However, the mobility files generated are of a simple text format, which gives us the opportunity to manually intervene inside the files and make appropriate changes.

The structure of the mobility file is as follows. Every node is assigned with its initial $\mathrm{X}, \mathrm{Y}, \mathrm{Z}$ coordinates in a command line. For example: at 0.0 (time) node(0) 2.3454 .1230 .0

After all nodes are assigned initial coordinates, setdest randomly selects the time point where each node will change its direction and speed in order to reach a specific $(X, Y, Z)$ point inside the topology. An example of such a command line is:

at 3.4567 (time) node (0) 4.89913 .75610 .392

Where the first parameter after "node(0)" (4.899) is the $\mathrm{X}$ coordinate for the reaching point, the second parameter (13.756) is the Y coordinate for the reaching point and the third parameter (10.392) is the speed of the mobile node. We have not included other parameters that are of no significance for the movement of the nodes in our examples.

We will explain how our mechanism works using a simple example. Let us consider the case where we want to create the topology presented in Figure 4.

The nodes need to move inside their own half of the network. The fact that there is limitation of movement using borders helps to keep a balanced percentage of differentiation. Simulation results are not affected, as the traffic generated is not unicast or multicast but broadcast. Our main goal is for all the mobile nodes to receive the broadcast packet

In the above topology, $20 \%$ of the mobile nodes $(4 / 20)$ are placed inside the right part of the network and $80 \%$ of them (16/20) are placed inside 


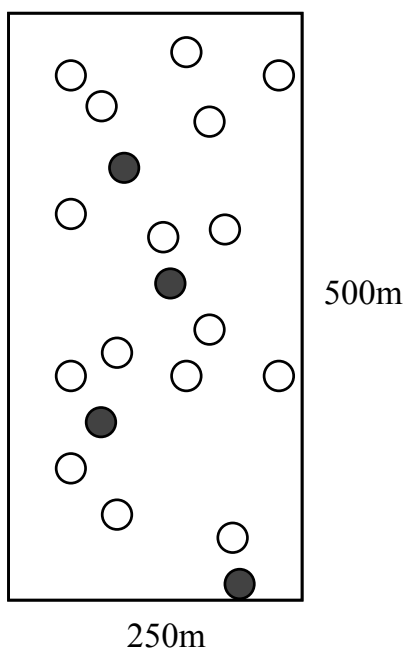

Figure 5. Base topology.

the left part. In order to create this topology, we need to start from a base topology as presented in Figure 5.

The volume of diversity is then specified by selecting an appropriate percentage of the mobile nodes, which in our example is $20 \%$. These are the black nodes of Figure 5. We developed a simple software tool that scans the mobility file for all the command lines that either initialize or change the movement of all 4 black nodes. The value of $X$ in these command lines is then increased by $250 \mathrm{~m}$, in order to migrate the black nodes over to a topology of identical length and width that touches the base topology vertically. Figure 6 shows the migration process.

The movement of the black nodes initially is limited with regards to the $\mathrm{X}$ coordinate between $0 \mathrm{~m}$ and $250 \mathrm{~m}$. Thus, after the modification of the mobility file, these nodes are restrained to move inside the right half of the topology, with the $\mathrm{X}$ value varying between $250 \mathrm{~m}$ and $500 \mathrm{~m}$.

As a result of the process described above we get the end result of Figure 4.

\section{PERFORMANCE ANALYSIS}

We implemented Distance-based Adaptive scheme (DibA) and Distance-based scheme (DB) using the network simulator NS2.30. We have used the NS2 code for DB provided by [9], [11].

\section{Simulation Set-up and Parameters}

Node mobility is simulated using mobility files that are generated by the NS2 mobility generation feature sedest. Our experiments make use of both normal and diverse topologies, in order to cover the majority of possible scenarios. The network area is of fixed size $500 \times 500 \mathrm{~m}^{2}$. The mobility files are created with zero pause time. Mobile nodes move with maximum speed of $5 \mathrm{~m} / \mathrm{sec}$. Each simulation

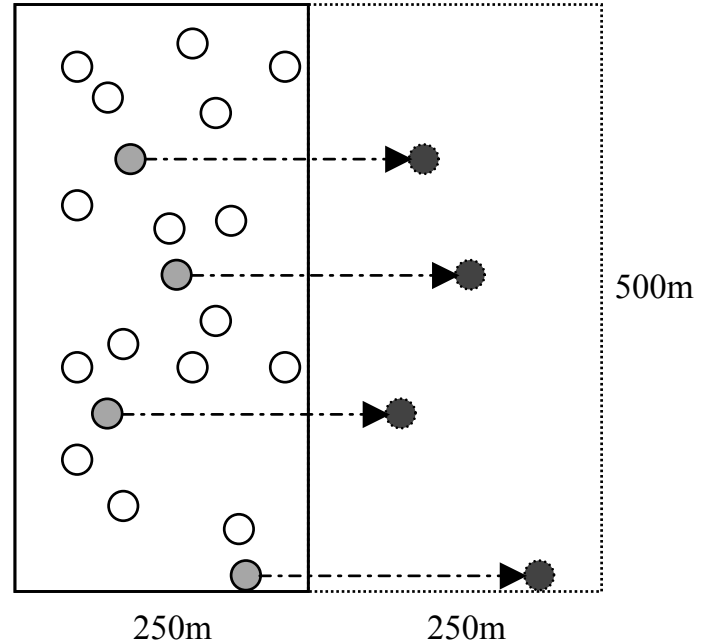

Figure 6. Migration process.

has duration of 100 secs and all mobile nodes use a transmission range of $100 \mathrm{~m}$.

Each scenario is restricted to the transmission of broadcast traffic only. This is a common strategy, especially when using very high broadcast generation rates (BGR). Combining normal traffic with broadcast traffic is a step further for our work with the implementation currently taking place. In order to avoid anomalies, we run three simulations for every scenario using three different mobility files. Our research has found no work until this point, where more than 3 or 4 repetitions are used. The final results are created as an average of the three simulations.

Experiments where performed using 3 different distance thresholds for DB of $10 \mathrm{~m}, 50 \mathrm{~m}$ and $90 \mathrm{~m}$, to cover the two extremes and an intermediate value. DibA tunes the distance threshold to one of the 3 thresholds mentioned above, depending on the local level of density. The number of nodes has a starting value of 20 and reaches a maximum of 200 nodes with a step of $20(20,40,60, \ldots, 200)$.

We first divide our simulations into two groups according to the broadcast generation rate. BGR is set to 5 packets $/ \mathrm{sec}$ and 60 packets $/ \mathrm{sec}$. Furthermore, we also divide the simulations depending on whether a normal or a diverse topology is used.

The following performance metrics are considered:

- Reachability - The percentage of nodes that successfully receive the broadcast message.

- Delay - The time elapsed from the initiation of the broadcast process until no more rebroadcasts take place.

- Average number of Packets transmitted per node (APT) - This is a self explained performance metric which is closely related to energy efficiency. 


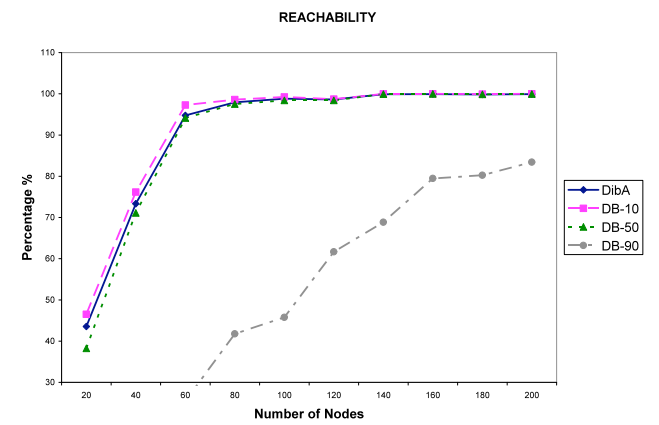

Figure 7. Reachability - normal - BGR 5p/s

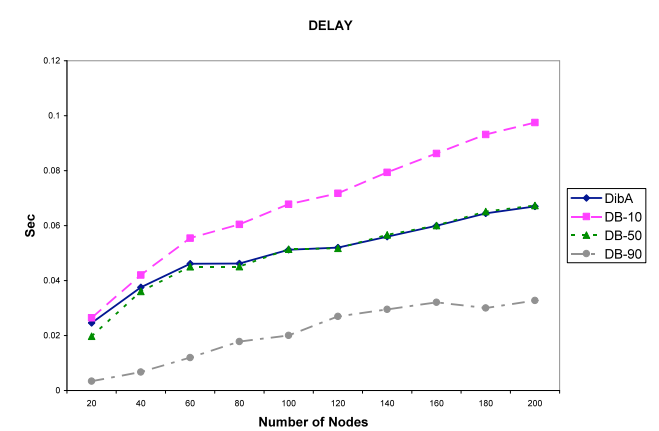

Figure 8. Delay - normal - BGR 5p/s

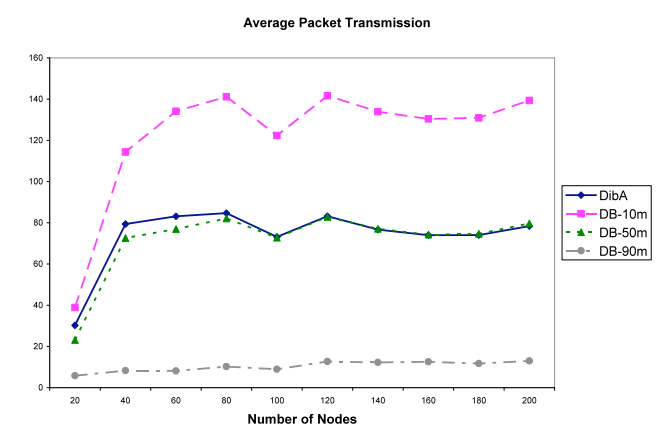

Figure 9. APT - normal - BGR 5p/s

\section{Simulation Results}

Figures 7, 8 and 9 present the performance of the 4 schemes, when normal scenarios are used and BGR is set to 5 packets/sec.

Figure 7 shows that DB-90 performs very poorly due to the high threshold value, whereas all the other schemes perform almost identical. Although DB-90 appears to be very fast in Figure 8, that is because of the very low level of reachability. DB10 is the slowest, despite the fact that has similar reachability with DB-50 and DibA. The latter two again perform in a similar way. Figure 9 shows that DB-10 uses a significantly higher number of transmissions in order to achieve the same level of reachability with DB-50 and DibA. Thus, it is the least energy efficient.

Figures 10,11 and 12 show how the 4 schemes perform when the topology is diverse and the broadcast generation rate is low.

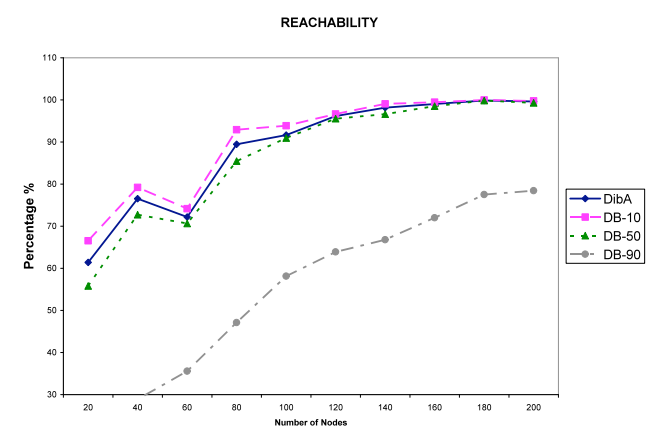

Figure 10. Reachability - diverse - BGR 5p/s

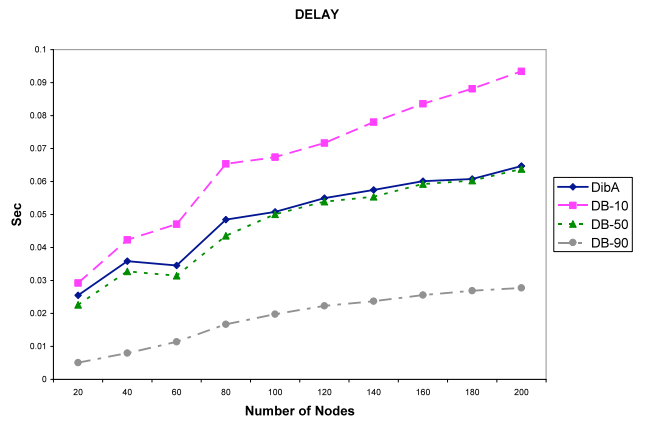

Figure 11. Delay - diverse - BGR 5p/s

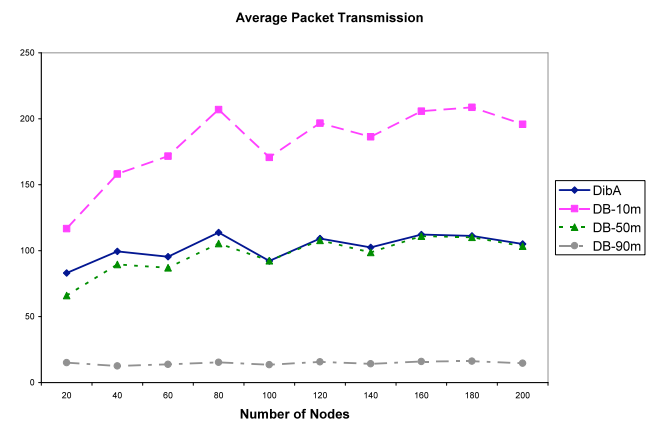

Figure 12. APT - diverse - BGR 5p/s

Figure 10 reflects the performance of all schemes in terms of reachability. Although DibA, DB-10 and DB-50 perform almost identical when the network is dense (120 nodes or more), for sparse topologies DB-10 is slightly better than DibA and in turn that is better than DB-50. DB-90 again performs poorly. DB-10's slightly better performance for reachability, proves to be extremely costly, as it is much slower than the rest and APT is almost double than the following scheme. Energy efficiency is very poor in these conditions. DibA appears to be better than DB-50 for sparse topologies and similar when density increases. Better reachability usually comes with more latency and more APT. For DibA and DB-50 this is reflected in figures 11 and 12. 


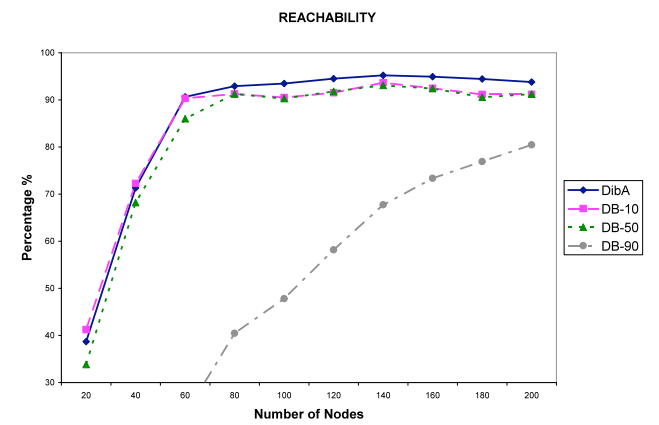

Figure 13. Reachability - normal - BGR 60p/s

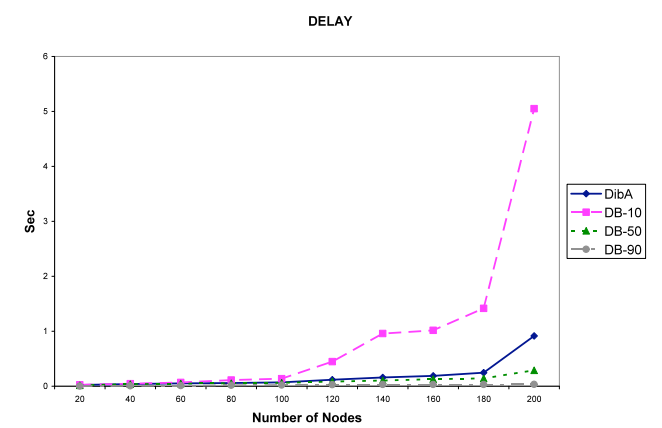

Figure 14. Delay - normal - BGR 60p/s

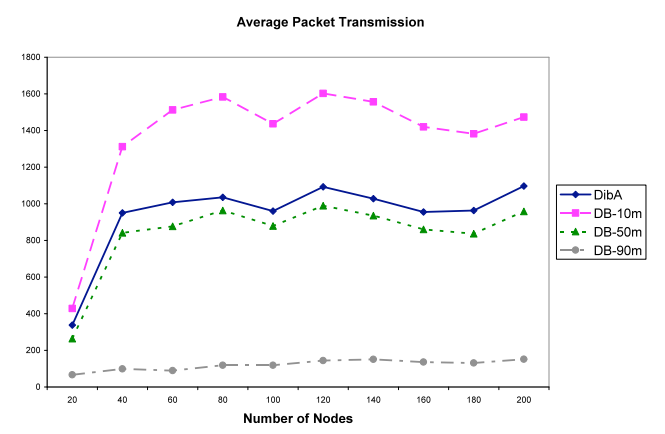

Figure 15. APT - normal - BGR 60p/s

Figures 13, 14 and 15 present the performance of the 4 schemes when normal scenarios are used and BGR is set to 60packets/sec.

Figure 13 shows that for sparse networks (up to 60 nodes) DibA and DB-10 have the same performance with DB-50 being slightly worse. For very dense networks, DibA is now performing better than the rest. DB-90 is completely outperformed. Despite the fact that DB-10 has lower reachability when compared to DibA, figures 14 and 15 show that it is disproportionally slower and energy inefficient. DB-50 shows slightly better performance for delay and APT, but that is due to its lower reachability.

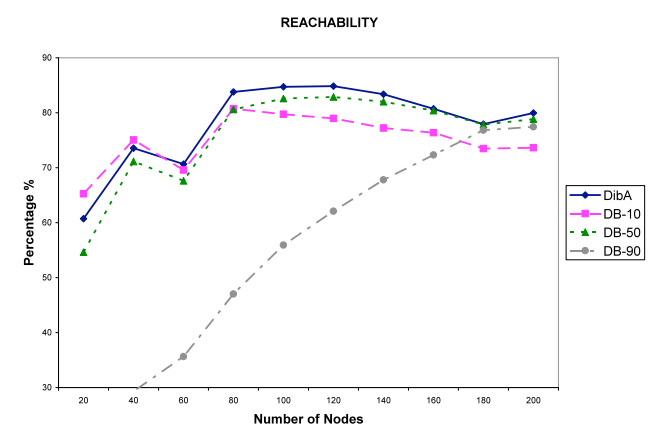

Figure 16. Reachability - diverse - BGR 60p/s

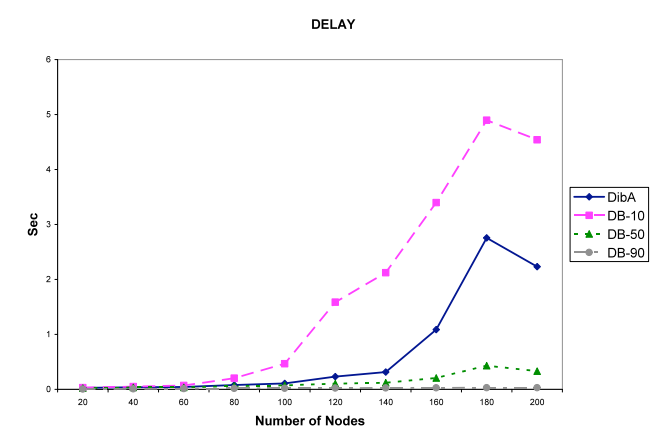

Figure 17. Delay - diverse - BGR 60p/s

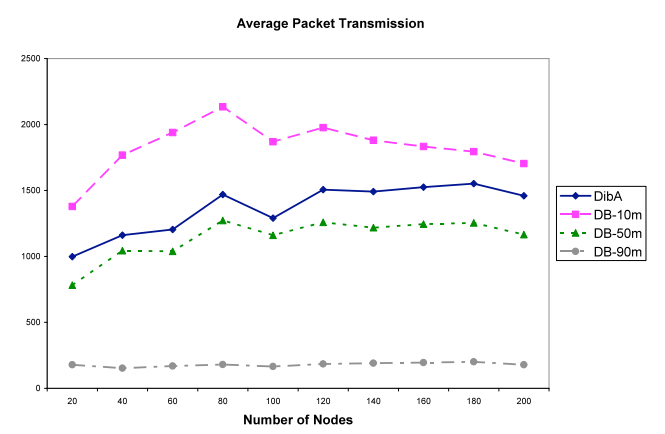

Figure 18. APT - diverse - BGR 60p/s

Figures 16, 17 and 18 show how the 4 schemes perform when the topology is diverse and the broadcast generation rate is extremely high.

In this group of experiments we have used a very high broadcast generation rate and extremely diverse network topologies. The results, in terms of reachability, are reflected in Figure 16. DB-10 is better for sparse networks, but as density increases, it is found to finish last for dense networks of 200 nodes. DB-50 proves to be more stable, but at no point does it perform better than all the rest. The results for DB-90, prove that even the use of a very low distance threshold is the appropriate selection when both density and traffic rate are set to high values. DibA appears to be the most reliable option. Figures 17 and 18 show that DB-10 is neither fast nor energy efficient. DB-50 performs well but being faster and more energy efficient is the result of its low reachability levels. 


\section{CONCLUSIONS}

In this paper we have shown how the use of static thresholds for the Distance-based scheme is not appropriate for all network topologies. For sparse networks with low broadcast generation rate, a low distance threshold is required. Respectively, when dealing with dense networks with high traffic rate, high distance thresholds offer a better performance.

The adaptive schemes proposed so far introduce further constraints as they either use GPS or increase overhead by exchanging HELLO packets. We presented our proposed scheme (DibA), which locally determines the density of the network and adjusts the distance threshold of every rebroadcasting operation without relying on HELLO packets or GPS systems.

The performance of DibA has been compared against those of DB using 3 different distance thresholds of $10 \mathrm{~m}, 50 \mathrm{~m}$ and $90 \mathrm{~m}$ studied under various other operational conditions throughout simulation. In particular, an extremely diverse network topology has also been used in order to demonstrate the advantages of adaptive schemes more appropriately.

It has been demonstrated that DibA outperforms DB for various topologies and broadcast generation rates. In addition, it is also more reliable and power efficient than DB.

\section{REFERENCES}

[1] D. Cavin, Y. Sasson, and A. Schiper, "On the Accuracy of MANET Simulators," POMC, 2002.

[2] K. Viswanath, and K Obraczka, "Modeling the performance of flooding in wireless multihop ad hoc networks," Computer Communications 29, 949-956, 2006.

[3] S. Ni, Y. Tseng, Y. Chen, and J. Sheu, "The broadcast storm problem in a mobile ad hoc network," MOBICOM, 1999.

[4] S. Leng, L. Zhang, L. Wu Yu, and C. Heng Tan, "An efficient broadcast relay scheme for MANETs," Computer Communications 28, 467-476, 2004.

[5] C. Zhu, M. J. Lee, and T. Saadawi, "A Border-aware broadcast scheme for Wireless Ad Hoc Networks," IEEE Explore, 2004.

[6] A. Qayyum, L. Viennot, and A. Laouiti, "Multipoint relaying for flooding broadcast messages in mobile wireless networks," Computer Society, 2002.

[7] C. Hsu, C. Chen, and H. Wang, "DISCOUNT: A Hybrid Probability-Based Broadcast Scheme for Wireless Ad Hoc Networks", IEEE, 2005.

[8] R. Purtoosi, H. Taheri, A. Mohammadi and F. Foroozan, "Improving broadcast performance by traffic isolation in wireless ad hoc networks", Int. J. Commun. Syst.; 19:10291043, 2006.

[9] B. J. Barritt, B. Malakooti, Z. Guo, "Intelligent Multiple-Criteria Broadcasting in Mobile Ad-hoc Networks", IEEE LCN / P2MNet, 2006.

[10] N. Bauer, M. Colagrosso, T. Camp, "Efficient implementations of all-to-all broadcasting in mobile ad hoc networks", Pervasive and Mobile Computing 1 311-342, 2005.

[11] B. Williams, and T. Camp, "Comparison of broadcast techniques for mobile ad hoc networks," MOBIHOC, 2002.

[12] Young-Ching Deng, Ching-Chi Shue, and Ferng-Ching lin, "An Adaptive Medium Access Control Protocol for Reliable Broadcast and Unicast in Ad Hoc Networks," IEICE - Tran. on Inf. \& Sys, 2006.

[13] Jung-Pil Ryu, Min-Su Kim, Sung-Ho Hwang, and Ki-Jun Han, "An Adaptive Probabilistic Broadcast Scheme for Ad-Hoc Networks," HNSMC, 2004

[14] Sung-Hee Lee and Young-Bae Ko, “An Efficient Neighbor Knowledge Based Broadcasting for Mobile Ad Hoc Networks," ICCS, 2006.

[15] Xiaohu Chen, Michalis Faloutsos, Srikanth Krishnamurthy, "Distance ADaptive (DAD) Broadcasting for Ad Hoc Networks," MILCOM, 2002.

[16] Michael D. Colagrosso, "Intelligent Broadcasting in Mobile Ad Hoc Networks: Three Classes of Adaptive Protocols," EURASIP, 2007.

[17] Xiaohu Chen, Michalis Faloutsos, Srikanth V. Krishnamurthy, "Power Adaptive Broadcasting with Local Information in Ad hoc networks," ICNP, 2003.

[18] Pradeep Kyasanur, Romit Roy Choudhury, Indranil Gupta, "Smart Gossip: An Adaptive Gossip-based Broadcasting Service for Sensor Networks," MAAS, 2006.

[19] Yu-Chee Tseng, Sze-Yao Ni, and En-Yu Shih, "Adaptive Approaches to Relieving Broadcast Storms in a Wireless Multihop Mobile Ad Hoc Network," IEEE Transactions on Computers, 2003. 